\title{
Does imbalance in chest X-ray datasets produce biased deep learning approaches for COVID-19 screening?
}

\author{
Lorena Alvarez-Rodríguez \\ Universidade da Coruña \\ Joaquim de Moura ( $\boldsymbol{\sim}$ joaquim.demoura@udc.es ) \\ Universidade da Coruña \\ Jorge Novo \\ Universidade da Coruña \\ Marcos Ortega \\ Universidade da Coruña
}

\section{Research Article}

Keywords: CAD system, Chest X-ray, COVID-19 screening, Data analysis, Deep learning

Posted Date: October 11th, 2021

DOl: https://doi.org/10.21203/rs.3.rs-960883/v1

License: (c) (i) This work is licensed under a Creative Commons Attribution 4.0 International License.

Read Full License 


\section{RESEARCH}

\section{Does imbalance in chest X-ray datasets produce biased deep learning approaches for COVID-19 screening?}

Lorena Álvarez-Rodríguez ${ }^{1,2}$, Joaquim de Moura ${ }^{1,2^{*}}$, Jorge Novo ${ }^{1,2}$ and Marcos Ortega ${ }^{1,2}$

\footnotetext{
*Correspondence:

joaquim.demoura@udc.es

${ }^{1}$ Centro de Investigación CITIC, Universidade da Coruña, Campus de Elviña, 15071, A Coruña, Spain

${ }^{2}$ Grupo VARPA, Instituto de

Investigación Biomédica de $A$

Coruña (INIBIC), Universidade da

Coruña, 15006, A Coruña, Spain

Full list of author information is

available at the end of the article
}

\begin{abstract}
Background: The health crisis resulting from the global COVID-19 pandemic highlighted more than ever the need for rapid, reliable and safe methods of diagnosis and monitoring of respiratory diseases. To study pulmonary involvement in detail, one of the most common resources is the use different lung imaging modalities (like chest radiography) to explore the possible affected areas.
\end{abstract}

Methods: In this work, we performed a comprehensive analysis of sex and age factors in chest X-ray images. The study of these recurrent patient characteristics in pathologies of this type is crucial, since there is a clear scarcity of data that may lead to biases when trying to develop systems that are as representative as possible, as well as to gain knowledge of the disease itself. To identify possible biases, we analyzed 3 different computational approaches for automatic COVID-19 screening: Normal vs COVID-19, Pneumonia vs COVID-19 and Non-COVID-19 vs COVID-19. The presented study was validated using two public chest $\mathrm{X}$-ray datasets, allowing a reliable analysis to support the clinical decision-making process in the context of this dramatic global pandemic.

Results: The obtained results for the sex-related imbalance analysis indicate that this factor slightly affects the system performance in the Normal VS COVID-19 and Pneumonia VS COVID-19 approaches, although the identified differences are not relevant enough to worsen considerably the system's response. Regarding the age-related imbalance analysis, this factor was observed to be again influencing the system in a more consistent way than the sex factor, as it was present in all the approaches. Once again, this worsening is not a major problem for our data and system, as it is not of great magnitude.

Conclusions: Multiple studies have been conducted in other fields in order to determine if certain patient characteristics such as sex or age influenced these deep learning systems. However, to the best of our knowledge, this study has not been done for COVID-19 despite the urgency and lack of COVID-19 chest x-ray images. The presented results evidenced that the proposed methodology and tested approaches allow a robust and reliable analysis to support the clinical decision-making process in this pandemic scenario.

Keywords: CAD system; Chest X-ray; COVID-19 screening; Data analysis; Deep learning

\section{Background}

In March 2020, the World Health Organization (WHO) declared the COVID-19 outbreak a pandemic. This highly contagious disease caused by the Severe Acute 
Respiratory Syndrome Coronavirus 2 (SARS-CoV-2) overwhelmed the healthcare system of many countries, forcing them to take drastic measurements to control the incessant flow of infected patients such as lockdown, curfew, among others health measures. This health crisis resulting from the global COVID-19 pandemic caused more than 219 million confirmed cases and more than 4.55 million deaths worldwide, highlighting more than ever the necessity of rapid, reliable and safe methods of diagnosing and monitoring respiratory diseases. COVID-19 is a demonstration of the impact that these diseases can have on society, with direct repercussions on public health and the global economy. Due to its particularities, these diseases present a very high transmission rate, as they can be easily transmitted by air. In this context, early detection and assessment of the evolution of patients with these diseases is vital, since many of them in their most severe phases, can lead to symptoms including acute respiratory failure, requiring the use of assisted breathing systems or admission to an intensive care unit (ICU).

In order to study lung involvement in detail, one of the most common resources is to use different lung imaging modalities (such as chest X-ray) to explore the possible affected areas. This requires a detailed analysis to identify and characterize the different pathological structures on the chest X-ray image, which should be performed by a professional with many years of experience. In this sense, the need to have a set of computational methodologies that allow detailed analysis of a chest $\mathrm{X}$-ray image for diagnostic purposes is critical, especially in the current pandemic scenario. As reference, Figure 1 shows 3 representative examples of chest X-ray images for 3 different scenarios: normal (patient without pulmonary conditions), patient with pneumonia (others than COVID-19) and patient with COVID-19.

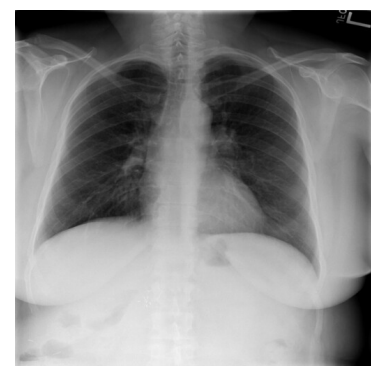

(a) Normal

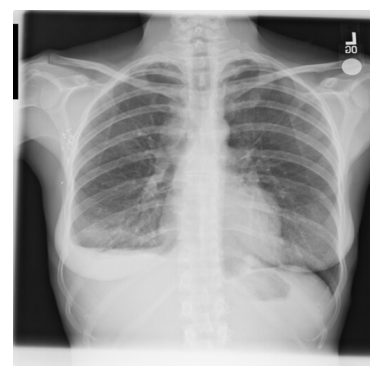

(b) Pneumonia

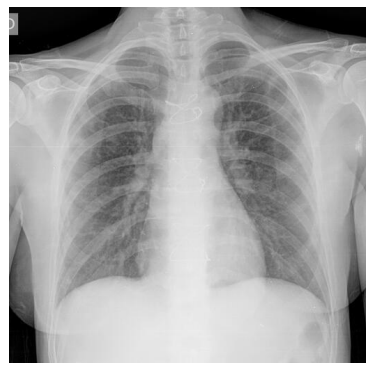

(c) COVID-19

Figure 1: Representative examples of chest X-ray images of normal (patient without pulmonary conditions), patient with pneumonia (others than COVID-19) and patient with COVID-19.

Given the great relevance of this topic, different authors have developed methodologies to support the diagnosis of COVID-19 using X-ray imaging [1, 2]. As reference, Wang et al. [3] developed an open access customized convolutional neural network (CNN) that detects COVID-19 signs in chest X-ray images. Along with this system, they also provided a public dataset named COVIDx that combines images from the main COVID-19 public datasets. In the work of Hammoudi et al. [4], the author proposed a deep learning system that distinguished bacterial pneumonia from viral pneumonia which could be caused by COVID-19. COVIDX-Net is a framework 
presented by Hemdan et al. [5] whose purpose is organizing seven different chest X-ray classifiers in order to diagnose COVID-19. In the work of Zhang et al. [6], the authors used Confidence Aware Anomaly Detection (CAAD) models to differentiate viral pneumonia from non-viral pneumonia and non-infected patients. Ozturk et al. [7] designed the DarkCovidNet, a deep learning architecture based on DarkNet, and their work was validated by a radiologist who reviewed heatmaps that showed where their system was identifying anomalies related to COVID-19. Shelke et al. [8] proposed a methodology that classified chest X-ray into normal, pneumonia, tuberculosis and COVID-19 classes, being able to rate severity. Yoo et al. [9] proposed a methodology based on classification trees that categorized X-ray images between normal and anomalies, and COVID-19 and non-COVID-19, respectively. In the work of Li et al. [10], the authors made predictions about a COVID-19 infected patient outcome by using a Siamese convolutional neural network [11] to estimate the disease severity. They used chest X-ray images to prognosticate patient's intubation or death, which is a useful resource for hospital resources management. De Moura et al. [12] presented 3 complementary approaches based on Dense Convolutional Network architectures specifically designed for the classification of chest X-ray images into normal, pathological and COVID-19. Waheed et al. [13] addressed the lack of COVID-19 chest X-ray and they tried to solve this by developing CovidGAN, a model based on Auxiliary Classifier Generative Adversarial Network that generates synthetic COVID-19 images. In the work of Morís et al. [14], the authors proposed a strategy to improve the performance of COVID-19 screening [15] by using 3 CycleGAN architectures to generate synthetic images from portable chest X-ray devices.

Nowadays, there is no doubt that deep learning methods are useful resources in the field of medical image analysis. However, these methods require a large amount of data for the developed systems to be used in a real scenario. This problem is known as data scarcity and exists even for more researched and common diseases, such as cancer or pneumonia, whose public datasets are scarce and, some of them, unbalanced, containing only certain types of patients. For instance, the Kaggle Pneumonia dataset [16] that was widely used in the development of different systems for automatic COVID-19 screening only contains pediatric chest X-ray images. This problem was commented by Cirillo et al. [17] in their work, as they describe how biased systems produce discriminatory results in the medical field. They focus on the sex and gender factors, as they consider these aspects to affect diseases, risks, treatments, symptoms, etc. In the work of Larrazabal et al. [18], the authors analysed how imbalance related to gender slightly biases deep learning systems when diagnosing some lung pathologies and abnormalities through chest X-ray images, even though observed worsening was not large. In the work of Vidal et al. [19], the authors proposed a methodology that attempts to alleviate this data scarcity problem in the COVID-19 domain by a two-step knowledge transfer to obtain a robust system able to segment lung regions from portable X-ray devices despite the scarcity of samples and lesser quality. However, to date, to the best of our knowledge, no such study, specifically for sex and age, has been performed for COVID-19 despite all the advances, number of articles and studies, the urgency and lack of COVID-19 chest-x ray images.

Therefore, in this work, we performed a comprehensive analysis of sex and age factors in the COVID-19 datasets. As mentioned above, these characteristics might 
influence the diagnosis of a disease of this type, where there is a clear problem of data scarcity, which may take us away from the goal of having systems that are as representative as possible and gaining more knowledge about the pathology itself. By thoroughly studying these patient characteristics, we made sure to answer the question of whether these factors produce bias in COVID-19 deep learningbased systems. For this purpose, we analyzed 3 different computational approaches for COVID-19 screening using chest X-ray images: (I) Normal vs COVID-19, (II) Pneumonia vs COVID-19 and (III) Non-COVID-19 vs COVID-19. The proposed study was validated using two state-of-the-art datasets publicly available to the scientific community.

This paper is organized as follows: Section "Materials and methods" describes the resources and deep learning approaches employed for the analysis of sex and age factors in the COVID-19 datasets; Section "Results" presents the obtained results; and finally, Sections "Discussion" and "Conclusion" conclude the manuscript, discussing the results and their impact in relation to the state of the art.

\section{Methods}

Datasets

In this section, we describe the 2 public chest X-ray datasets used for this research: (I) HM Hospitals COVID-19 dataset "Covid data saves lives" and (II) RSNA Pneumonia Challenge dataset. Both are described in detail below.

\section{HM Hospitals COVID-19 dataset}

HM Hospitals made available to the scientific community an anonymous dataset with all clinical information of patients treated in their hospitals by the COVID-19 virus [20]. This dataset is available upon request and must be approved by the HM Hospitals Research Ethics Committee. It consists of 2310 patients with a diagnosis of "COVID-19 positive" or "COVID-19 pending" admitted to HM Hospitals. Chest $\mathrm{X}$-rays are available for some of the patients, and these were taken during the time they were hospitalized. In this sense, we used 5493 posteroanterior chest X-ray images from 1862 different patients whose age and sex are distributed as indicated in Figure 2 for our COVID-19 class.

\section{RSNA Normal/Pneumonia dataset}

The RSNA Pneumonia Challenge dataset [21] is a subset of the ChestX-ray8 dataset [22] created for the Kaggle challenge on the MD.ai platform in collaboration with the Radiological Society of North America (RSNA). This dataset consists of 16248 $\mathrm{X}$-ray images, considering only the posteroanterior chest view, resulting in 9452 images for normal cases and 6796 images for patients diagnosed with pneumonia. In this dataset, we also have information about the age and sex of the patients. These characteristics are distributed in our subset as indicated in Figure 3 for normal and pneumonia cases.

\section{Software and Hardware resources}

In this work, we used Python (version 3.6.6) for the implementation of the conducted studies and machine learning libraries PyTorch (version 0.4.1) and Scikit-learn 


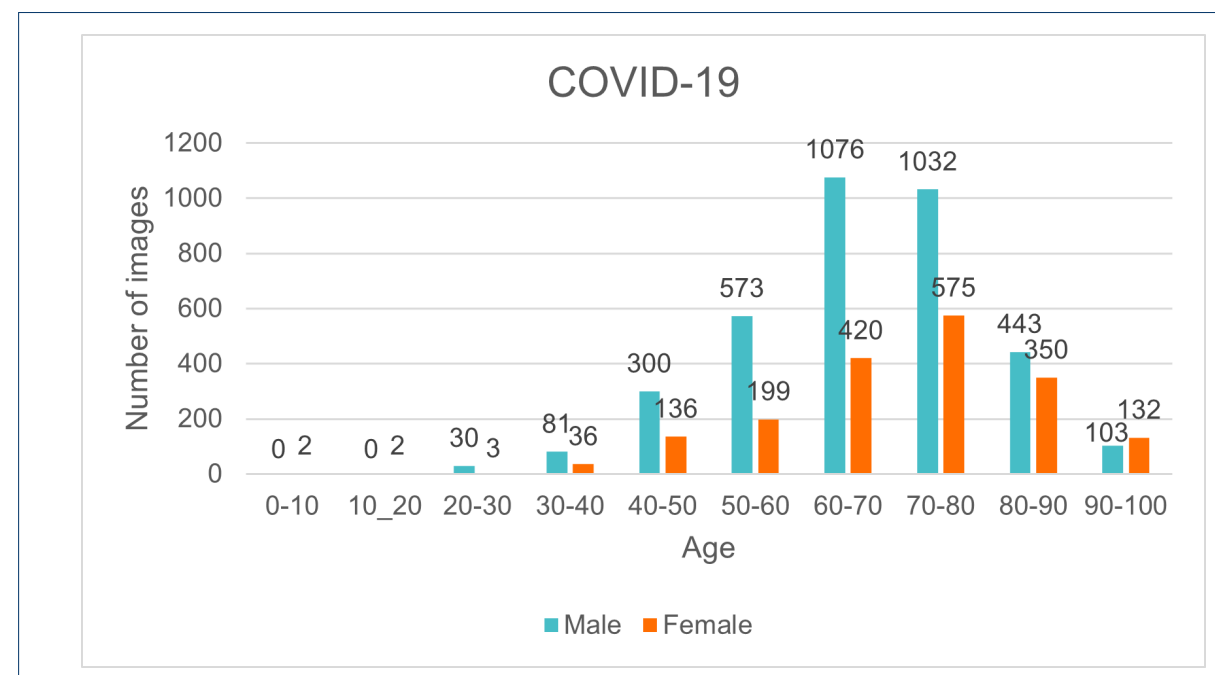

Figure 2: Age and sex distribution for chest X-ray images of the HM Hospitals COVID-19 dataset.

(version 0.24.2) were used to train, validation and test the obtained models, as well as to get the metrics of their performances.

In addition, in order to facilitate the replication of our studies, we present in Table 1 the main specifications of the hardware used to perform the experiments.

\begin{tabular}{|c|c|}
\hline Name & Description \\
\hline $\begin{array}{r}\text { OS } \\
\text { Kernel } \\
\text { Architecture }\end{array}$ & $\begin{array}{l}\text { DEBIAN GNU/Linux } 10 \\
\text { Linux } 4.18 .0-2 \text {-amd64 } \\
\times 86-64\end{array}$ \\
\hline $\begin{array}{r}\text { CPU } \\
\text { Motherboard } \\
\text { RAM } \\
\text { HDD }\end{array}$ & $\begin{array}{l}\text { Intel(R) Xeon(R) CPU E5-2650 v4 @ } 2.20 \mathrm{GHz} \\
\text { Lenovo NeXtScale n×360 M5 } \\
16 \text { GB de RAM GDDR5 } \\
\text { IBM ServeRAID M5210 } 930 \mathrm{~GB}\end{array}$ \\
\hline $\begin{array}{l}\text { GPU } \\
\text { Driver Version } \\
\text { CUDA Version }\end{array}$ & $\begin{array}{l}\text { NVIDIA Tesla P100 } \\
396.44 \\
9.2\end{array}$ \\
\hline
\end{tabular}

Table 1: Specifications of the equipment used throughout the project to carry out the experiments.

\section{Architecture}

In this work, we exploited the potential of the DenseNet-161 architecture [23]. This architecture is composed of dense blocks linked by transition layers, which in turn are formed by convolution and pooling layers. These dense blocks have layers with their own feature maps which consists of a batch normalisation operation, a ReLu operation and a $3 \times 3$ convolution with $k$ filters, where $k$ is the growth rate. Each of them receives the feature maps of all the previous layers, so that the collective knowledge of all the predecessor layers is preserved. In our case, this growth ratio $k$ is 48 , and the depth of the architecture $L$ is 161 . However, we modified its original structure to support the binary output defined in our computational approaches, as depicted in Table 2. This architecture provided satisfactory results in similar works aimed at classifying chest X-rays of patients with COVID-19 [15, 12, 14], which led us to choose it for this work. 


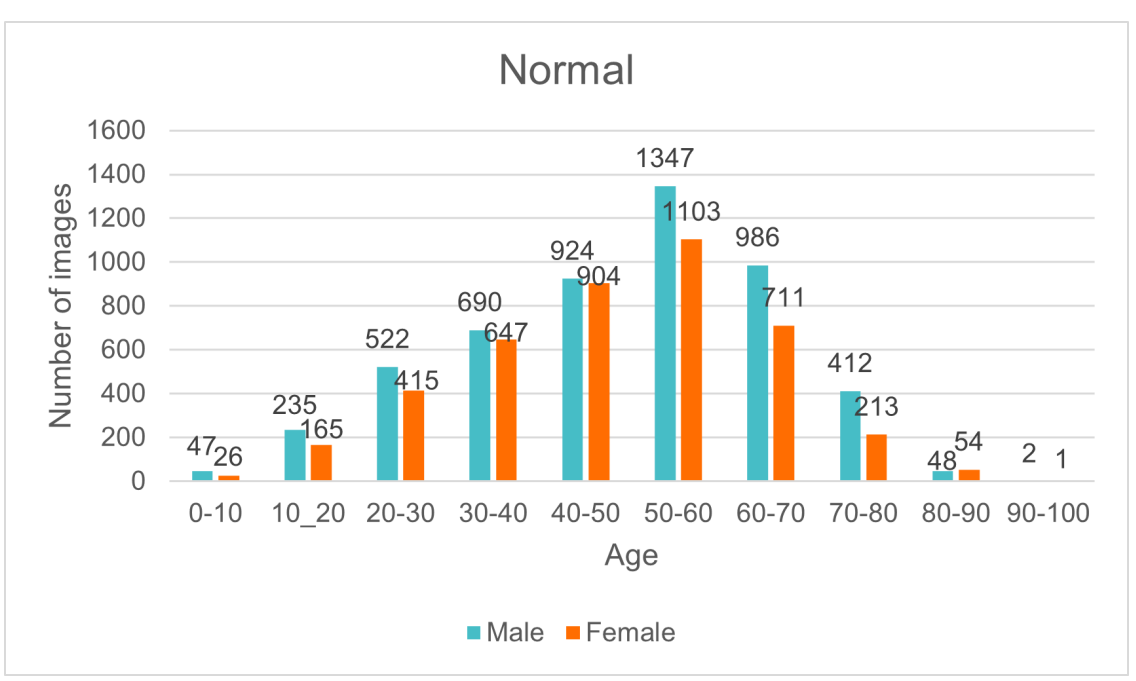

(a) Normal

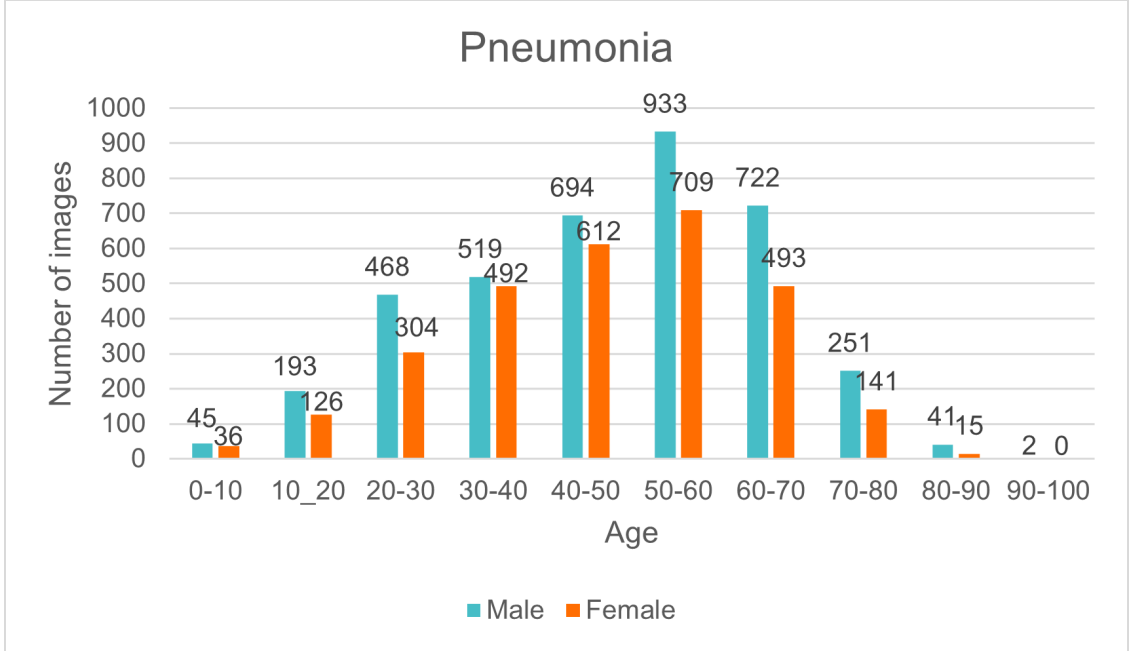

(b) Pneumonia

Figure 3: Age and sex distribution for chest X-ray images of the RSNA dataset. 


\begin{tabular}{|c|c|c|c|}
\hline Layers & Output size & \multicolumn{2}{|c|}{ DenseNet-161 } \\
\hline Convolution & $112 \times 112$ & \multicolumn{2}{|c|}{ Conv. $7 \times 7$, stride 2} \\
\hline Pooling & $56 \times 56$ & \multicolumn{2}{|c|}{ Max pool $3 \times 3$, stride 2} \\
\hline Dense block (1) & $56 \times 56$ & $\begin{array}{ll}1 \times 1 & \text { conv } \\
3 \times 3 & \text { conv }\end{array}$ & $\times 6$ \\
\hline Trancition laver (1) & $56 \times 56$ & \multicolumn{2}{|l|}{ Conv. $1 \times 1$} \\
\hline Irdinsicion Idyer (I) & $28 \times 28$ & \multicolumn{2}{|c|}{$2 \times 2$ average pool, stride 2} \\
\hline Dense block (2) & $28 \times 28$ & $\begin{array}{ll}1 \times 1 & \text { conv } \\
3 \times 3 & \text { conv }\end{array}$ & $\times 12$ \\
\hline Troncition laver (2) & $28 \times 28$ & \multicolumn{2}{|l|}{ Conv. $1 \times 1$} \\
\hline Iransition layer (2) & $14 \times 14$ & \multicolumn{2}{|c|}{$2 \times 2$ average pool, stride 2} \\
\hline Dense block (3) & $14 \times 14$ & $\begin{array}{ll}1 \times 1 & \text { conv } \\
3 \times 3 & \text { conv }\end{array}$ & $\times 36$ \\
\hline & $14 \times 14$ & \multicolumn{2}{|l|}{ Conv. $1 \times 1$} \\
\hline Iransition layer (3) & $7 \times 7$ & \multicolumn{2}{|c|}{$2 \times 2$ average pool, stride 2} \\
\hline Dense block (4) & $7 \times 7$ & $\begin{array}{ll}1 \times 1 & \text { conv } v \\
3 \times 3 & \text { conv }\end{array}$ & $\times 24$ \\
\hline Classification laver & $1 \times 1$ & \multirow{2}{*}{\multicolumn{2}{|c|}{$7 \times 7$ global average pool }} \\
\hline & & & \\
\hline
\end{tabular}

Table 2: DenseNet-161 adapted structure.

\section{Computational approaches for screening tasks}

As illustrated in Figure 4, we present 3 different approaches which classify X-ray images into 2 categories to differentiate COVID-19 patients from certain types of patients, as normal and pneumonia ones. Each of these approaches will be explained in more detail below, but in general these 3 different approaches cover a wide range of scenarios in which we can study in depth how gender and age factors affect the diagnosis of COVID-19 in deep learning systems. In this way, we will be able to draw more solid and contrasted conclusions, as most of the cases where a COVID-19 screening task is performed are taken into account and a bias could be more clearly detected.

$1^{\text {st }}$ Approach: Normal vs. COVID-19

In this first scenario, we trained a model to obtain a consolidated approach to distinguish between normal cases (control patients without lung conditions but who may have other systemic pathologies) and COVID-19. We consider this scenario to be very useful as it is realistic and complex, as it is more difficult than distinguishing only between healthy patients and COVID-19. Moreover, this approach is present in the literature [24]. Both the fact that it is a situation that can occur in a clinical context and that it is a case that can be widely found in the state of the art make the casuistry present in this approach interesting when studying the influence of our target factors.

$2^{\text {nd }}$ Approach: Pneumonia vs. COVID-19

Given the similarities between COVID-19 and both viral and bacterial pneumonia, this second approach aims to differentiate between patients with COVID-19 and patients with pneumonia not caused by COVID-19. Thus, 2 different categories are predicted: pneumonia and COVID-19. Similar approaches have been studied in related works $[8,25]$. Again, this is a complex situation that could be found in a real screening task and it is broadly studied in the state of art as well, so we find here a number of interesting cases where to explore the impact that sex and age could have. 

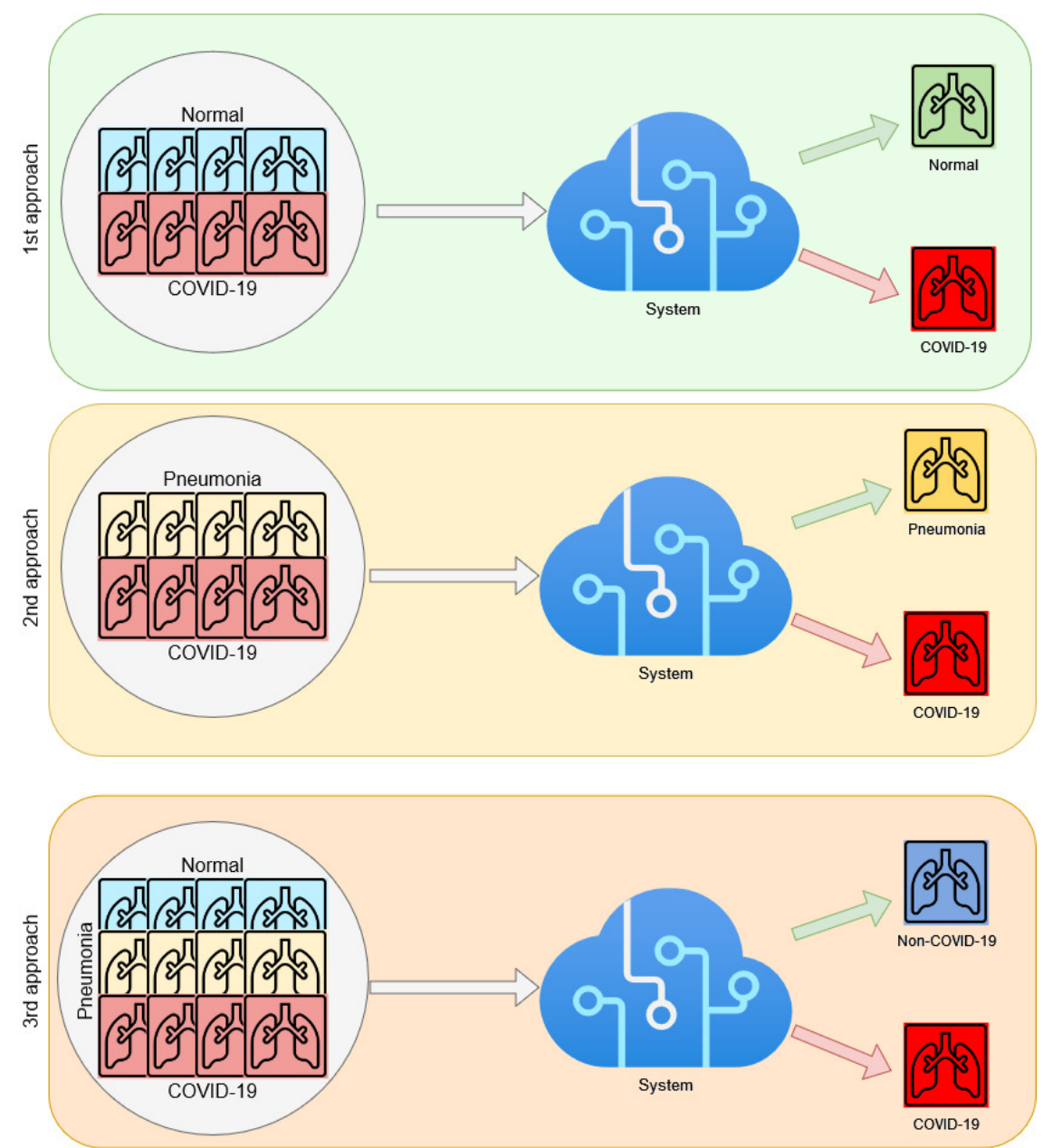

Figure 4: Schematic representation of computational approaches for COVID-19 screening using X-ray images.

\section{$3^{\text {rd }}$ Approach: Non-COVID-19 vs. COVID-19}

In this third approach, two categories are considered: one that has normal and pneumonia patients, named Non-COVID-19, and another one that has only COVID19 patients. In this way, we can analyse the degree of separability between COVID-19 patients from all other cases. This kind of approach is common in related works $[3,5,26]$. Thus, this approach allows us, again, to investigate how our target factors could affect a wide number of real and complex cases taken into account here.

\section{Training Details}

The final dataset for each experiment where we will study the sex and age factors was divided into mutually exclusive subsets, being $(60 \%, 20 \%$, and $20 \%)$ for training, validation, and testing, respectively. Regarding the training, we started from the DenseNet-161 model pre-trained with the ImageNet [27] dataset, making use of the transfer learning strategy, but modifying the output layer to adapt it to our specific classification problem. In this way, the training process will be more efficient due to the faster convergence of the training and validation curves. It also reduces the 
number of labeled images necessary for the process to be adequate [19]. On the other hand, a cross-entropy loss function is performed on the output class and the ground truth for the target X-ray image. The optimization during the training is carried out by Stochastic Gradient Descent (SGD) [28] with a learning rate constant of 0.01, a mini-batch size of 4 , and a first-order momentum of 0.9 , all of them obtained by exhaustive experimentation. This optimiser has proven to be very efficient, despite its simplicity, for the discriminative learning of classifiers under convex loss functions, defined as follows, where $Y$ represents the ground truth values and $\hat{Y}$ represents the estimated values for each identified category:

$$
L=-Y \cdot \log (\hat{Y})
$$

A complete training epoch includes a run through all the samples of the training set. Each training process had 200 epochs, since a larger number of epochs would not produce of epochs did not produce significant improvements neither in the loss function nor in the accuracy metrics. In addition, to ensure the generalization capability of the approaches presented, each experiment was repeated 5 times independently of each other with random sample selection, so it was necessary to calculate the means of these repetitions to evaluate the overall global performance. To compensate for the lack of available X-ray images and thus avoid problems of overfitting and to increase the generalization capacity, data augmentation was performed to obtain more robust and stable models. Thus, scaling and horizontal rotation operations were performed, which are appropriated given the symmetrical nature of the chest X-ray image, so the variability of the data used was increased. We consider this configuration to be suitable enough for our sex and age study, as it has provided satisfactory results in similar works [15, 14, 12].

\section{Evaluation}

The performance of the presented computational approaches was evaluated by comparing the predictions provided by the models with the ground truth labels annotated in the X-ray image datasets. Then, the values of True Positives (TP), True Negatives (TN), False Positives (FP) and False Negatives (FN) were considered to calculate different metrics that are commonly used in the literature $[15,14,12]$ to assess the stability of computational methods for medical imaging problems. Following the reference of these similar works, we also decided to use these metrics for our analysis of the sex and age factors. Thus, Precision, Recall, F1-score, and Accuracy were calculated for each approach as follows.

$$
\begin{aligned}
& \text { Precision }=\frac{T P}{T P+F P} \\
& \text { Recall }=\frac{T P}{T P+F N} \\
& F 1-\text { score }=2 * \frac{\text { Precision } * \text { Recall }}{\text { Precision }+ \text { Recall }}
\end{aligned}
$$




$$
\text { Accuracy }=\frac{T P+T N}{T P+T N+F P+F N}
$$

\section{Results}

In this section, we present the experimental results of the proposed computational approaches for the classification of COVID-19 in chest X-ray images, covering a wide range of cases that will allow us to draw more contrasted and solid conclusions regarding the studied factors of sex and age. In particular, we perform two different and complementary studies on the COVID-19 dataset. The first one analyses the influence of the sex factor for each of the 3 approaches: (I) Normal VS COVID-19, (II) Pneumonia VS COVID-19 and (III) Non-COVID-19 VS COVID-19. The second one performs a similar analysis, but in this case considering patients by age ranges. Both studies are described below.

\section{Sex-related imbalance analysis}

One of the main characteristics of a patient that can influence a diagnostic system is sex $[17,18]$. Especially in chest x-rays, we might think that differences in size, in addition to other typical sex characteristics such as the presence of breasts, could imply taking the images in different postures or certain abnormalities in the samples that could be mistaken for signs of a pathology, in this case this being COVID-19 [29]. In Figure 5, we exemplify these differences with 2 patients of different sexes who have COVID-19. Considering how important is to identify a bias related to the sex of the patient, we designed the following study in order to test whether this characteristic influences diagnosing COVID-19.

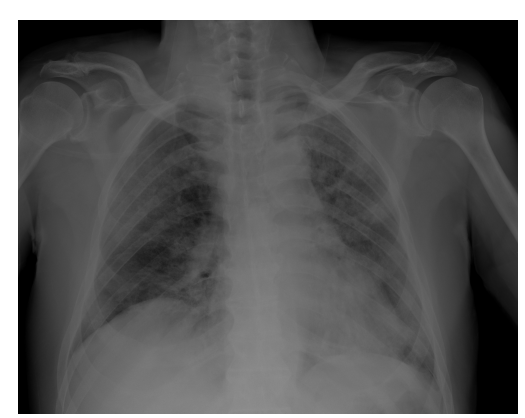

(a) Male

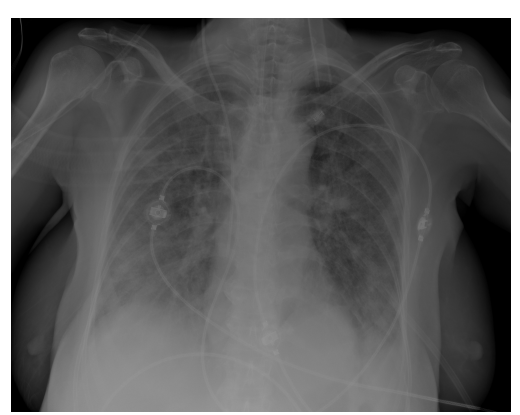

(b) Female

Figure 5: Example of two representative chest X-ray images of male and female patients diagnosed with COVID-19.

In this first analysis, we explored intermediate imbalance scenarios in which female and male patients diagnosed with COVID-19 were analysed in different proportions with $10 \%$ intervals, ranging from $0 \%$ male patients and $100 \%$ female patients to $100 \%$ male patients and $0 \%$ female patients. Thus, we conducted a comprehensive analysis with 11 different configurations for each computational approach. For each imbalance case, we get a model that is then tested using the remaining images not used during training. Afterwards, we compare the results obtained for each scenario 
with our baseline (50\% female and 50\% male). Regarding the amount of images considered for each approach, we used 700 COVID-19 images from 700 different patients. To maintain balance between this COVID-19 class and the other classes, 700 X-ray images were randomly selected and divided according to the sex of the patient, as indicated in Table 3. Therefore, each of the 11 experiments was performed using 1400 chest X-ray images.

\begin{tabular}{|c|c|c|}
\hline Approach & Normal & Pneumonia \\
\hline Normal VS COVID-19 & $350 \mathrm{M}+350 \mathrm{~F}$ & 0 \\
\hline Pneumonia VS COVID-19 & 0 & $350 \mathrm{M}+350 \mathrm{~F}$ \\
\hline Non-COVID-19 VS COVID-19 & $175 \mathrm{M}+175 \mathrm{~F}$ & $175 \mathrm{M}+175 \mathrm{~F}$ \\
\hline
\end{tabular}

Table 3: Distribution of randomly selected X-ray images for each computational approach in the sex-related imbalance analysis.

Analysis of the $1^{\text {st }}$ approach: Normal vs. COVID-19

In Table 4 we present a comparative analysis of the performance at the test stage using precision, recall, and F1-score measures, where we highlight our baseline as we are going to use it to compare our metrics. As for the mean accuracy obtained at each scenario, our values ranged from $0.9757 \pm 0.0105$ at the $40 \% \mathrm{M} 60 \% \mathrm{~F}$ case, to $0.9835 \pm 0.0105$ at the $90 \% \mathrm{M} 10 \% \mathrm{~F}$ case. The standard deviation of these metrics was always below $2.1 \%$, being the highest at the $60 \% \mathrm{M} 40 \% \mathrm{~F}$ case, and the lowest at $30 \% \mathrm{M} 90 \% \mathrm{~F}$ with $0.58 \%$. In general, it can be observed that the differences between the metrics are small when compared to our baseline and their values are maintained regardless of the studied scenario.

\begin{tabular}{|c|c|c|c|c|}
\hline Experiment & Class & Precision & Recall & F1-Score \\
\hline \multirow{2}{*}{$\mathbf{0} \%$ M 100\%F } & Normal & $0.9872 \pm 0.0076$ & $0.9831 \pm 0.0124$ & $0.9851 \pm 0.0090$ \\
\cline { 2 - 5 } & COVID-19 & $0.9827 \pm 0.0132$ & $0.9869 \pm 0.0080$ & $0.9848 \pm 0.0095$ \\
\hline \multirow{2}{*}{$\mathbf{1 0 \% M ~ 9 0 \% F}$} & Normal & $0.9827 \pm 0.0185$ & $0.9882 \pm 0.0082$ & $0.9854 \pm 0.0092$ \\
\cline { 2 - 5 } & COVID-19 & $0.9888 \pm 0.0079$ & $0.9833 \pm 0.0181$ & $0.9859 \pm 0.0090$ \\
\hline \multirow{2}{*}{$\mathbf{2 0 \% M ~ 8 0 \% F}$} & Normal & $0.9957 \pm 0.0063$ & $0.9821 \pm 0.0139$ & $0.9888 \pm 0.0078$ \\
\cline { 2 - 5 } & COVID-19 & $0.9810 \pm 0.0154$ & $0.9956 \pm 0.0065$ & $0.9882 \pm 0.0085$ \\
\hline \multirow{2}{*}{$\mathbf{3 0 \% M ~ 7 0 \% F}$} & Normal & $0.9830 \pm 0.0113$ & $0.9800 \pm 0.0112$ & $0.9814 \pm 0.0053$ \\
\cline { 2 - 5 } & COVID-19 & $0.9800 \pm 0.0117$ & $0.9827 \pm 0.0121$ & $0.9813 \pm 0.0063$ \\
\hline \multirow{2}{*}{$\mathbf{4 0 \% M ~ 6 0 \% F}$} & Normal & $0.9839 \pm 0.0120$ & $0.9670 \pm 0.0121$ & $0.9754 \pm 0.0108$ \\
\cline { 2 - 5 } & COVID-19 & $0.9677 \pm 0.0115$ & $0.9843 \pm 0.0115$ & $0.9759 \pm 0.0102$ \\
\hline \multirow{2}{*}{$\mathbf{5 0 \% M ~ 5 0 \% F}$} & Normal & $0.9902 \pm 0.0104$ & $0.9817 \pm 0.0095$ & $0.9859 \pm 0.0077$ \\
& COVID-19 & $0.9813 \pm 0.0093$ & $0.9896 \pm 0.0112$ & $0.9854 \pm 0.0082$ \\
\hline \multirow{2}{*}{$\mathbf{6 0 \% M ~ 4 0 \% F}$} & Normal & $0.9818 \pm 0.0198$ & $0.9874 \pm 0.0240$ & $0.9846 \pm 0.0208$ \\
\cline { 2 - 5 } & COVID-19 & $0.9868 \pm 0.0257$ & $0.9811 \pm 0.0213$ & $0.9839 \pm 0.0224$ \\
\hline \multirow{2}{*}{$\mathbf{7 0 \% M ~ 3 0 \% F}$} & Normal & $0.9928 \pm 0.0100$ & $0.9803 \pm 0.0175$ & $0.9865 \pm 0.0130$ \\
\cline { 2 - 5 } & COVID-19 & $0.9800 \pm 0.0188$ & $0.9927 \pm 0.0103$ & $0.9863 \pm 0.0139$ \\
\hline \multirow{2}{*}{$\mathbf{8 0 \% M ~ 2 0 \% F}$} & Normal & $0.9843 \pm 0.0135$ & $0.9843 \pm 0.0128$ & $0.9842 \pm 0.0083$ \\
\cline { 2 - 5 } & COVID-19 & $0.9843 \pm 0.0123$ & $0.9843 \pm 0.0135$ & $0.9842 \pm 0.0081$ \\
\hline \multirow{2}{*}{$\mathbf{9 0 \% M ~ 1 0 \% F}$} & Normal & $0.9843 \pm 0.0135$ & $0.9957 \pm 0.0063$ & $0.9899 \pm 0.0090$ \\
\cline { 2 - 5 } & COVID-19 & $0.9955 \pm 0.0065$ & $0.9845 \pm 0.0136$ & $0.9899 \pm 0.0094$ \\
\hline \multirow{2}{*}{$\mathbf{1 0 0 \% M ~ 0 \% F}$} & Normal & $0.9826 \pm 0.0139$ & $0.9840 \pm 0.0091$ & $0.9833 \pm 0.0104$ \\
\cline { 2 - 5 } & COVID-19 & $0.9844 \pm 0.0095$ & $0.9831 \pm 0.0138$ & $0.9837 \pm 0.0107$ \\
\hline
\end{tabular}

Table 4: Mean \pm standard deviation of the results obtained in the test stage for the classification of chest X-ray images between Normal VS COVID-19 after 5 independent repetitions. The baseline is highlighted in grey.

Analysis of the $2^{\text {nd }}$ approach: Pneumonia vs. COVID-19

The second group of experiments deals with the analysis of sex-related imbalance in the second approach. In this line, Table 5 show a comparative analysis of the 
performance at the test stage using precision, recall, and F1-score measures. Here, we highlight our baseline as we are going to use it to compare our metrics. As we can see, the results show a similar tendency to the previous set of experiments of the first approach, with values for the mean accuracy ranged from $0.9721 \pm 0.0187$ at the $0 \% \mathrm{M} 100 \% \mathrm{~F}$ case, to $0.9892 \pm 0.0091$ at the $100 \% \mathrm{M} 0 \% \mathrm{~F}$ case. The standard deviation of these metrics was always below $1.8 \%$, being the highest at the $0 \% \mathrm{M}$ $100 \% \mathrm{~F}$ case, and the lowest at $10 \% \mathrm{M} 90 \% \mathrm{~F}$ with $0.86 \%$.

\begin{tabular}{|c|c|c|c|c|}
\hline Experiment & Class & Precision & Recall & F1-Score \\
\hline \multirow{2}{*}{ 0\%M 100\%F } & Pneumonia & $0.9769 \pm 0.0174$ & $0.9675 \pm 0.0224$ & $0.9721 \pm 0.0191$ \\
\cline { 2 - 5 } & COVID-19 & $0.9671 \pm 0.0220$ & $0.9771 \pm 0.0167$ & $0.9720 \pm 0.0184$ \\
\hline \multirow{2}{*}{$\mathbf{1 0 \% M ~ 9 0 \% F}$} & Pneumonia & $0.9838 \pm 0.0166$ & $0.9756 \pm 0.0116$ & $0.9796 \pm 0.0090$ \\
\cline { 2 - 5 } & COVID-19 & $0.9761 \pm 0.0118$ & $0.9848 \pm 0.0155$ & $0.9803 \pm 0.0082$ \\
\hline \multirow{2}{*}{ 20\%M 80\%F } & Pneumonia & $0.9830 \pm 0.0216$ & $0.9898 \pm 0.0110$ & $0.9864 \pm 0.0157$ \\
\cline { 2 - 5 } & COVID-19 & $0.9899 \pm 0.0109$ & $0.9829 \pm 0.0214$ & $0.9864 \pm 0.0155$ \\
\hline \multirow{2}{*}{$30 \% M \mathbf{7 0 \% F}$} & Pneumonia & $0.9815 \pm 0.0089$ & $0.9815 \pm 0.0125$ & $0.9815 \pm 0.0096$ \\
\cline { 2 - 5 } & COVID-19 & $0.9813 \pm 0.0133$ & $0.9812 \pm 0.0102$ & $0.9812 \pm 0.0108$ \\
\hline \multirow{2}{*}{$\mathbf{4 0 \% M ~ 6 0 \% F}$} & Pneumonia & $0.9897 \pm 0.0065$ & $0.9854 \pm 0.0116$ & $0.9875 \pm 0.0076$ \\
\cline { 2 - 5 } & COVID-19 & $0.9860 \pm 0.0108$ & $0.9902 \pm 0.0062$ & $0.9881 \pm 0.0071$ \\
\hline \multirow{2}{*}{$\mathbf{5 0 \% M ~ 5 0 \% F}$} & Pneumonia & $0.9830 \pm 0.0107$ & $0.9766 \pm 0.0206$ & $0.9797 \pm 0.0135$ \\
& COVID-19 & $0.9751 \pm 0.0223$ & $0.9825 \pm 0.0109$ & $0.9787 \pm 0.0143$ \\
\hline \multirow{2}{*}{$\mathbf{6 0 \% M ~ 4 0 \% F}$} & Pneumonia & $0.9856 \pm 0.0088$ & $0.9779 \pm 0.0191$ & $0.9817 \pm 0.0119$ \\
\cline { 2 - 5 } & COVID-19 & $0.9766 \pm 0.0212$ & $0.9855 \pm 0.0086$ & $0.9810 \pm 0.0131$ \\
\hline \multirow{2}{*}{$\mathbf{7 0 \% M ~ 3 0 \% F}$} & Pneumonia & $0.9868 \pm 0.0121$ & $0.9854 \pm 0.0145$ & $0.9861 \pm 0.0130$ \\
\cline { 2 - 5 } & COVID-19 & $0.9859 \pm 0.0140$ & $0.9874 \pm 0.0114$ & $0.9866 \pm 0.0124$ \\
\hline \multirow{2}{*}{$\mathbf{8 0 \% M ~ 2 0 \% F}$} & Pneumonia & $0.9816 \pm 0.0094$ & $0.9778 \pm 0.0151$ & $0.9797 \pm 0.0094$ \\
\cline { 2 - 5 } & COVID-19 & $0.9766 \pm 0.0170$ & $0.9811 \pm 0.0095$ & $0.9788 \pm 0.0104$ \\
\hline \multirow{2}{*}{$\mathbf{9 0 \% M ~ 1 0 \% F}$} & Pneumonia & $0.9769 \pm 0.0174$ & $0.9675 \pm 0.0224$ & $0.9721 \pm 0.0191$ \\
\cline { 2 - 5 } & COVID-19 & $0.9671 \pm 0.0220$ & $0.9771 \pm 0.0167$ & $0.9720 \pm 0.0184$ \\
\hline \multirow{2}{*}{$\mathbf{1 0 0 \% M ~ 0 \% F}$} & Pneumonia & $0.9769 \pm 0.0174$ & $0.9675 \pm 0.0224$ & $0.9721 \pm 0.0191$ \\
\cline { 2 - 5 } & COVID-19 & $0.9671 \pm 0.0220$ & $0.9771 \pm 0.0167$ & $0.9720 \pm 0.0184$ \\
\hline
\end{tabular}

Table 5: Mean \pm standard deviation of the results obtained in the test stage for the classification of chest X-ray images between Pneumonia VS COVID-19 after 5 independent repetitions. The baseline is highlighted in grey.

Analysis of the $3^{\text {rd }}$ approach: Non-COVID-19 vs. COVID-19

In this third set of experiments, we analyzed the behavior of the sex factor imbalance in the data on separability between the Non-COVID-19 vs. COVID-19 classes. Table 6 shows the results of the test stage in terms of precision, recall and F1-Score for each class, after performing the proposed experiments, and we highlighted our baseline as we are going to use it to compare our metrics. As we can see, these results reflect that all models are able to accurately separate samples from both classes. As for the mean accuracy obtained at each scenario, our values ranged from $0.9700 \pm 0.0117$ at the $40 \% \mathrm{M} 60 \% \mathrm{~F}$ case, to $0.9857 \pm 0.0035$ at the $100 \% \mathrm{M} 0 \% \mathrm{~F}$ case. The standard deviation of these metrics was always below $1.3 \%$, being the highest at the $60 \% \mathrm{M}$ $40 \% \mathrm{~F}$ case, and the lowest at $100 \% \mathrm{M} 0 \% \mathrm{~F}$ with $0.35 \%$.

\section{Age-related imbalance analysis}

Age-related deterioration of both the skeleton and the musculature of the body is visible on chest X-rays, which may affect the diagnosis obtained from them [17, 30]. In addition, older COVID-19 patients often require more medical equipment that appears on chest X-ray images, such as intravenous lines, ventilators, pacemakers, and so on, which may again affect the diagnosis obtained from the X-rays [29]. 


\begin{tabular}{|c|c|c|c|c|}
\hline Experiment & Class & Precision & Recall & F1-Score \\
\hline \multirow{2}{*}{$0 \% \mathrm{M} 100 \% \mathrm{~F}$} & Non-COVID-19 & $0.9813 \pm 0.0103$ & $0.9826 \pm 0.0167$ & $0.9819 \pm 0.0090$ \\
\hline & COVID-19 & $0.9832 \pm 0.0156$ & $0.9815 \pm 0.0108$ & $0.9823 \pm 0.0084$ \\
\hline \multirow{2}{*}{$10 \% \mathrm{M} 90 \% \mathrm{~F}$} & Non-CO & \pm 0.0093 & $0.9795 \pm 0.0196$ & $0.9781 \pm 0.0125$ \\
\hline & COV & 0.980 & $0.9715=$ & $0.9990 \pm$ \\
\hline \multirow{2}{*}{$20 \% \mathrm{M} 80 \% \mathrm{~F}$} & Non-COVID-19 & 0.9814 & 0.9900 & $0.9855 \pm$ \\
\hline & COVID-19 & 0.9899 & 0.9819 & 0.9858 \\
\hline \multirow{2}{*}{$30 \% \mathrm{M} 70 \% \mathrm{~F}$} & Non-COVID-19 & $0.9854 \pm 0$ & $38 \pm 0.0150$ & 0.9846 \\
\hline & COVID-19 & $0.9847 \pm 0.0142$ & $0.9859 \pm 0.0096$ & $0.9853 \pm 0.0112$ \\
\hline \multirow{2}{*}{$40 \% \mathrm{M} 60 \% \mathrm{~F}$} & Non-COI & $0.9680 \pm 0$ & $0.9707 \pm 0.0213$ & $0.9692 \pm($ \\
\hline & COVI & 0.97 & 0.9692 & $0.9706=$ \\
\hline \multirow{2}{*}{$50 \% \mathrm{M} \mathrm{50 \% F}$} & Non-CO & 0.990 & 0.9885 & $0.9893=$ \\
\hline & COVI & 0.977 & 0.9793 & $0.9782 \pm c$ \\
\hline \multirow{2}{*}{$60 \% \mathrm{M} 40 \% \mathrm{~F}$} & Non-COVID-19 & $0.9813 \pm 0$ & $0.9785 \pm 0$ & $0.9798 \pm c$ \\
\hline & COVID-19 & $0.9786 \pm 0.0116$ & $0.9816 \pm 0.0188$ & $0.9800 \pm 0.0134$ \\
\hline \multirow{2}{*}{$70 \% \mathrm{M} 30 \% \mathrm{~F}$} & Non-COV & $0.9782 \pm 0$ & $0.9896 \pm 0.0084$ & $0.9838 \pm 0.0062$ \\
\hline & COVID & 0.9903 & $0.9792 \pm 0.0146$ & $0.9846 \pm c$ \\
\hline \multirow{2}{*}{$80 \%$ M $20 \% \mathrm{~F}$} & Non-CC & 0.98 & 0.970 & 0.9760 \\
\hline & COVID & $0.9698 \pm 0$ & $0.9812 \pm 0.0230$ & $0.9753 \pm 0$ \\
\hline \multirow{2}{*}{$90 \% \mathrm{M} 10 \% \mathrm{~F}$} & Non-COVID-19 & $0.9813 \pm 0.0103$ & $0.9826 \pm 0.0167$ & $0.9819 \pm 0.0090$ \\
\hline & COVID-19 & $0.9832 \pm 0.0156$ & $0.9815 \pm 0.0108$ & $0.9823 \pm 0.0084$ \\
\hline \multirow{2}{*}{$100 \% \mathrm{M} 0 \% \mathrm{~F}$} & Non-COVID-19 & $0.9813 \pm 0.0103$ & $0.9826 \pm 0.0167$ & $0.9819 \pm 0.0090$ \\
\hline & COVID-19 & $0.9832 \pm 0.0156$ & $0.9815 \pm 0.0108$ & $0.9823 \pm 0.0084$ \\
\hline
\end{tabular}

Table 6: Mean \pm standard deviation of the results obtained in the test stage for the classification of chest X-ray images between Non-COVID-19 VS COVID-19 after 5 independent repetitions. The baseline is highlighted in grey.

To illustrate these characteristics associated with different ages, Figure 6 shows representative examples of different COVID-19 patients ranging in age from 47 to 93 years old. These differences raise the need for a detailed study of how the patient age affects the diagnosis of COVID-19. Therefore, we describe below the analysis we have carried out for this purpose.

For the age-related imbalance study, we defined 6 different age ranges: 0-40, 40-50, $50-60,60-70,70-80, \geq 80$. For each range, we used only images from patients in that age spectrum for training and then tested it with the remaining images. We analysed the differences between the age group used for training, which acts as our baseline, and all other ages. Regarding the exact number of samples used for each class in our 3 computational approaches, we present our distribution in Table 7. Using this amount of images of each class, we sought to emphasise the older age groups, who suffer more from the disease and have to go through a more critical diagnostic process, but also adapting to the number of samples we had available from the studied Normal, Pneumonia and COVID-19 classes of interest.

\begin{tabular}{|c|c|c|c|}
\hline Age & Normal VS COVID-19 & Pneumonia VS COVID-19 & Non-COVID-19 VS COVID-19 \\
\hline$<40$ & 154 vs 154 & 154 vs 154 & $(77+77)$ vs 154 \\
\hline $40-50$ & 436 vs 436 & 436 vs 436 & $(218+218)$ vs 436 \\
\hline $50-60$ & 772 vs 772 & 772 vs 772 & $(386+386)$ vs 772 \\
\hline $60-70$ & 1496 vs 1496 & 1215 vs 1215 & $(748+748)$ vs 1496 \\
\hline $70-80$ & 625 vs 625 & 392 vs 392 & $(392+392)$ vs 784 \\
\hline$\geq 80$ & 105 vs 105 & 58 vs 58 & $(58+58)$ vs 116 \\
\hline
\end{tabular}

Table 7: Number of samples of each class considered per approach.

In the following sections, we will show the results of our six baselines (one per age range) for each approach. However, the details of how these baselines responded to the different age groups will be discussed in the Discussion section in order to simplify this section and facilitate understanding. 


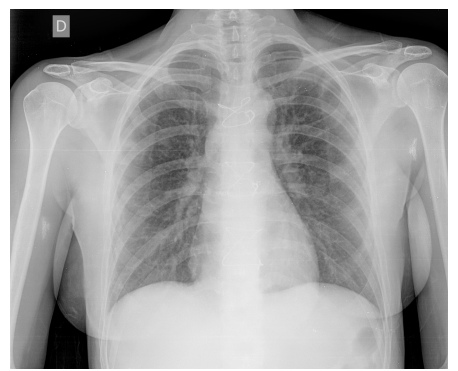

(a) 47 years old

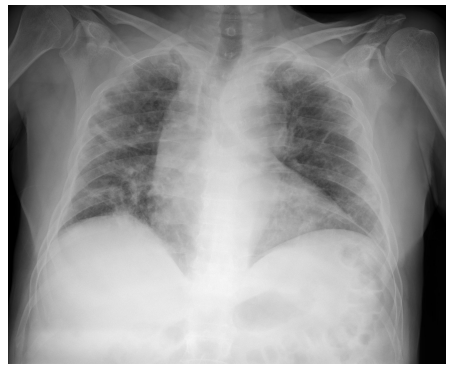

(c) 79 years old

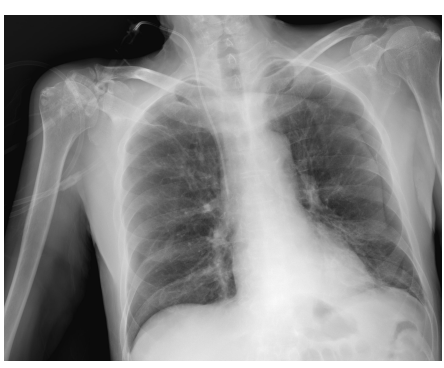

(b) 69 years old

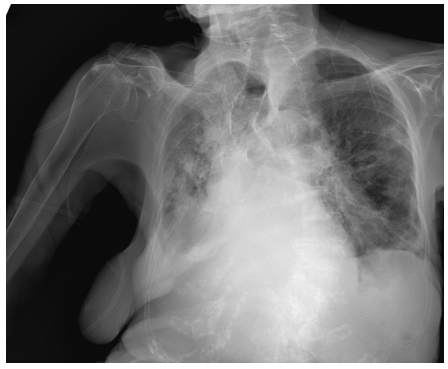

(d) 93 years old

Figure 6: Example of four representative chest X-ray images of patients of different ages diagnosed with COVID-19.

Analysis of the $1^{\text {st }}$ approach: Normal vs. COVID-19

For this first approach, we present in Table 8 precision, recall and F1-score means and their standard deviation obtained at test for each experiment training with only one age group. These results for our six baselines were satisfactory and mainly stable, as the metrics were over $90 \%$ in most cases and standard deviation was under $8 \%$. Regarding the mean accuracy obtained for each one of these baselines, we obtained the following values: $0.9587 \pm 0.0298,0.9748 \pm 0.0012,0.9877 \pm 0.0001,0.9876 \pm 0.0001$, $0.9808 \pm 0.0004$ and $0.9429 \pm 0.0086$, ordering them from the youngest to the oldest age group. In general, this indicates that our baselines are acceptable and stable, since the accuracy was above $94 \%$ and the standard deviation kept under $8.6 \%$.

\begin{tabular}{|c|c|c|c|c|}
\hline Exp. & Class & Precision & Recall & F1-Score \\
\hline \multirow{2}{*}{$<40$} & Normal & $0.9453 \pm 0.0514$ & $0.9749 \pm 0.0349$ & $0.9588 \pm 0.0277$ \\
\cline { 2 - 5 } & COVID-19 & $0.9742 \pm 0.0355$ & $0.9438 \pm 0.0588$ & $0.9573 \pm 0.0276$ \\
\hline \multirow{2}{*}{$40-50$} & Normal & $0.9673 \pm 0.0288$ & $0.9814 \pm 0.0141$ & $0.9742 \pm 0.0193$ \\
\cline { 2 - 5 } & COVID-19 & $0.9816 \pm 0.0125$ & $0.9693 \pm 0.0250$ & $0.9753 \pm 0.0156$ \\
\hline \multirow{2}{*}{$50-60$} & Normal & $0.9911 \pm 0.0057$ & $0.9846 \pm 0.0080$ & $0.9878 \pm 0.0053$ \\
\cline { 2 - 5 } & COVID-19 & $0.9844 \pm 0.0066$ & $0.9907 \pm 0.0058$ & $0.9875 \pm 0.0043$ \\
\hline \multirow{2}{*}{$60-70$} & Normal & $0.9925 \pm 0.0055$ & $0.9826 \pm 0.0062$ & $0.9875 \pm 0.0053$ \\
\cline { 2 - 5 } & COVID-19 & $0.9828 \pm 0.0064$ & $0.9926 \pm 0.0055$ & $0.9877 \pm 0.0054$ \\
\hline \multirow{2}{*}{$70-80$} & Normal & $0.9780 \pm 0.0171$ & $0.9846 \pm 0.0086$ & $0.9812 \pm 0.0102$ \\
\cline { 2 - 5 } & COVID-19 & $0.9832 \pm 0.0111$ & $0.9774 \pm 0.0173$ & $0.9802 \pm 0.0115$ \\
\hline \multirow{2}{*}{$\geq 80$} & Normal & $0.9373 \pm 0.0691$ & $0.8912 \pm 0.0849$ & $0.9125 \pm 0.0690$ \\
\cline { 2 - 5 } & COVID-19 & $0.8859 \pm 0.0805$ & $0.9255 \pm 0.0800$ & $0.9043 \pm 0.0734$ \\
\hline
\end{tabular}

Table 8: Mean \pm standard deviation of the results obtained in the test stage for the classification of chest X-ray images between Normal VS COVID-19 after 5 independent repetitions. 
Analysis of the $2^{\text {nd }}$ approach: Pneumonia vs. COVID-19

For our second set of experiments, we summarized in Table 9 the metrics and their standard deviation obtained for our baseline models at the test stage for each experiment training with only one age group. Again, these models had acceptable results, as they were above $90 \%$ in nearly all cases and its standard deviations were below $10 \%$. As for the the mean accuracy obtained for each one of these baselines, we obtained these values for every baseline ordered by age: $0.9396 \pm 0.0027$, $0.9760 \pm 0.0004,0.9800 \pm 0.0005,0.9919 \pm 0.0001,0.9772 \pm 0.0004$ and $0.9083 \pm 0.043$. Overall, these metrics are satisfactory and steady, being above $90 \%$ and with a standard deviation under $4.3 \%$.

\begin{tabular}{|c|c|c|c|c|}
\hline Exp. & Class & Precision & Recall & F1-Score \\
\hline \multirow{2}{*}{$<40$} & Pneumonia & $0.9603 \pm 0.0440$ & $0.9292 \pm 0.0241$ & $0.9438 \pm 0.0188$ \\
\cline { 2 - 5 } & COVID-19 & $0.9199 \pm 0.0303$ & $0.9498 \pm 0.0625$ & $0.9336 \pm 0.0356$ \\
\hline \multirow{2}{*}{$40-50$} & Pneumonia & $0.9811 \pm 0.0177$ & $0.9717 \pm 0.0161$ & $0.9762 \pm 0.0105$ \\
\cline { 2 - 5 } & COVID-19 & $0.9689 \pm 0.0219$ & $0.9821 \pm 0.0172$ & $0.9752 \pm 0.0122$ \\
\hline \multirow{2}{*}{$50-60$} & Pneumonia & $0.9802 \pm 0.0118$ & $0.9815 \pm 0.0116$ & $0.9808 \pm 0.0112$ \\
\cline { 2 - 5 } & COVID-19 & $0.9796 \pm 0.0126$ & $0.9784 \pm 0.0129$ & $0.9790 \pm 0.0122$ \\
\hline \multirow{2}{*}{$60-70$} & Pneumonia & $0.9942 \pm 0.0046$ & $0.9895 \pm 0.0036$ & $0.9918 \pm 0.0036$ \\
\cline { 2 - 5 } & COVID-19 & $0.9893 \pm 0.0040$ & $0.9944 \pm 0.0045$ & $0.9919 \pm 0.0035$ \\
\hline \multirow{2}{*}{$70-80$} & Pneumonia & $0.9869 \pm 0.0132$ & $0.9678 \pm 0.0101$ & $0.9772 \pm 0.0097$ \\
\cline { 2 - 5 } & COVID-19 & $0.9668 \pm 0.0123$ & $0.9874 \pm 0.0122$ & $0.9770 \pm 0.0097$ \\
\hline \multirow{2}{*}{$\geq 80$} & Pneumonia & $0.8850 \pm 0.1117$ & $0.9000 \pm 0.1732$ & $0.8893 \pm 0.1380$ \\
\cline { 2 - 5 } & COVID-19 & $0.9346 \pm 0.1084$ & $0.9095 \pm 0.0831$ & $0.9195 \pm 0.0840$ \\
\hline
\end{tabular}

Table 9: Mean \pm standard deviation of the results obtained in the test stage for the classification of chest X-ray images between Pneumonia VS COVID-19 after 5 independent repetitions.

Analysis of the $3^{\text {rd }}$ approach: Non-COVID-19 vs. COVID-19

Finally for this third approach, we show in Table 10 precision, recall and F1-score means and their standard deviation obtained at test for each experiment training with only one age group. Following the trend that we have already seen in the two previous approaches, our baseline models had adequate metrics, as they were above $90 \%$ in all scenarios and the corresponding standard deviation was below $6 \%$. The results obtained for the mean accuracy from the youngest to the oldest baseline were the following: $0.9683 \pm 0.0020,0.9760 \pm 0.0002,0.9819 \pm 0.0002,0.9913 \pm 0.8 \times 10^{-5}$, $0.9898 \pm 0.8 \times 10^{-4}$ and $0.9234 \pm 0.0077$. As we can see, all baselines remained above $96 \%$ and their standard deviation was under $7.7 \%$, which make these metrics satisfactory and mainly stable.

\section{Discussion}

Regarding the sex-related imbalance analysis, the precision, recall and F1-score measures shown in Results section were in every experiment in all the approaches above $96 \%$, which is a satisfactory result. As for accuracy, we summarized the obtained measures for every experiment for each approach in Figure 7. We can see here how there are no extreme peaks in either the accuracy or its standard deviation in none of the approaches, and differences between experiments and approaches are around 5\%. Although the Normal VS COVID-19 approach has a bigger standard deviation peak at the $60 \%$ male and $40 \%$ female experiment, all values remain closer and similar to our baseline. The same occurs for the Pneumonia VS COVID-19 


\begin{tabular}{|c|c|c|c|c|}
\hline Exp. & Class & Precision & Recall & F1-Score \\
\hline \multirow{2}{*}{$<40$} & Non-COVID-19 & $0.9754 \pm 0.0141$ & $0.9625 \pm 0.0344$ & $0.9688 \pm 0.0231$ \\
\cline { 2 - 5 } & COVID-19 & $0.9617 \pm 0.0352$ & $0.9725 \pm 0.0157$ & $0.9669 \pm 0.0226$ \\
\hline \multirow{2}{*}{$40-50$} & Non-COVID-19 & $0.9707 \pm 0.0155$ & $0.9821 \pm 0.0154$ & $0.9762 \pm 0.0059$ \\
\cline { 2 - 5 } & COVID-19 & $0.9812 \pm 0.0189$ & $0.9701 \pm 0.0184$ & $0.9754 \pm 0.0093$ \\
\hline \multirow{2}{*}{$50-60$} & Non-COVID-19 & $0.9849 \pm 0.0096$ & $0.9802 \pm 0.0105$ & $0.9825 \pm 0.0078$ \\
\cline { 2 - 5 } & COVID-19 & $0.9785 \pm 0.0123$ & $0.9840 \pm 0.0096$ & $0.9812 \pm 0.0084$ \\
\hline \multirow{2}{*}{$60-70$} & Non-COVID-19 & $0.9925 \pm 0.0043$ & $0.9898 \pm 0.0041$ & $0.9911 \pm 0.0016$ \\
\cline { 2 - 5 } & COVID-19 & $0.9901 \pm 0.0039$ & $0.9927 \pm 0.0043$ & $0.9914 \pm 0.0011$ \\
\hline \multirow{2}{*}{$70-80$} & Non-COVID-19 & $0.9950 \pm 0.0052$ & $0.9850 \pm 0.0069$ & $0.9900 \pm 0.0046$ \\
\cline { 2 - 5 } & COVID-19 & $0.9846 \pm 0.0072$ & $0.9947 \pm 0.0053$ & $0.9896 \pm 0.0047$ \\
\hline \multirow{2}{*}{$\geq 80$} & Non-COVID-19 & $0.9429 \pm 0.0547$ & $0.9107 \pm 0.0633$ & $0.9250 \pm 0.0426$ \\
\cline { 2 - 5 } & COVID-19 & $0.9068 \pm 0.0573$ & $0.9380 \pm 0.0695$ & $0.9206 \pm 0.0480$ \\
\hline
\end{tabular}

Table 10: Mean \pm standard deviation of the results obtained in the test stage for the classification of chest X-ray images between Non-COVID-19 VS COVID-19 after 5 independent repetitions.

approach, as accuracy continues to be stable and alike our baseline. In the NonCOVID-19 VS COVID-19 approach we have a slightly different scenario, since most of the obtained values are under our baseline, especially in experiments $40 \%$ male and $60 \%$ female, and $80 \%$ male and $20 \%$ female. Despite these differences, we can observe how accuracy remains stable and similar to other approaches. All these satisfactory results, together with the stability observed in all the scenarios considered in each of our approaches, indicate that this factor has not clearly affected the diagnosis offered by our system. If it had, we would have seen graphs with more evident differences between each of the different sex ratios with which we experimented. Thereby, no influence caused by the sex factor was observed. Although male and female patients may have differentiating features that allow us to identify their sex on chest x-rays, such as breasts, differences in shape and size, etc., these typically sex-associated features do not influence their COVID-19 diagnosis and do not favour one sex over the other, as they do not interfere with the lung assessment. For example, differences in shape and size do not difficult the finding of suspicious densities in the lung itself, and those densities related to the mammary glands are easily discarded, as they are present in most female patients and do not usually obscure COVID-19 related findings.

Regarding the age-related imbalance analysis, the precision, recall and F1-score measures shown in Results section were in every experiment in all approaches above $96 \%$, which is a satisfactory result. As for accuracy, we summarized the obtained results for each approach in Figure 8, taking as a reference the baselines metrics shown in the Results section. In this accuracy comparative across all six age ranges it is presented how its standard deviation increases as baseline patients get older than 70. The worst instability peaks are in the 70-80 range in the Normal VS COVID-19 approach and the $\geq 80$ range in the Pneumonia VS COVID-19 approach, but these increases only represent a worsening of $10 \%$. This behaviour is not as clearly observed for the Non-COVID-19 VS COVID-19 approach, since its standard deviations rises at the $\geq 80$ range, but not as noticeably as in other approaches. In relation to the accuracy metric itself, it is observed how the closer to the baseline age the tested age range gets, the better accuracies are obtained. However, these differences are not of great magnitude. In general, the third approach seems like the best and most stable of the three ones considered, since its accuracy is consistently 


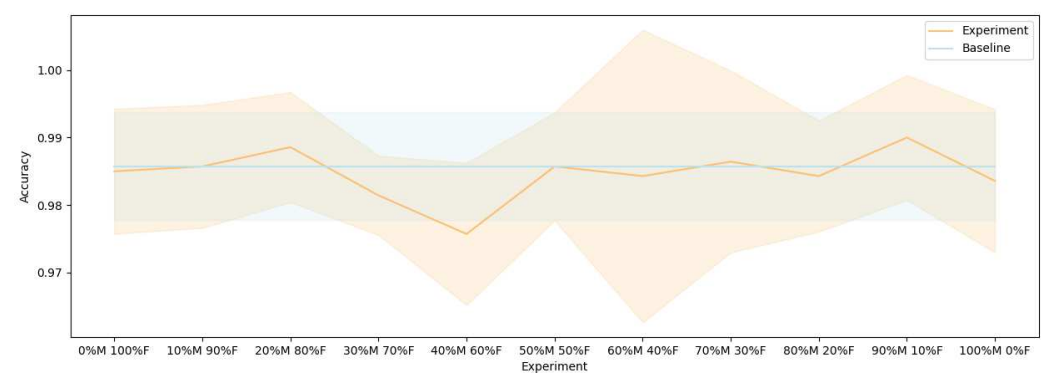

(a) Normal VS COVID-19

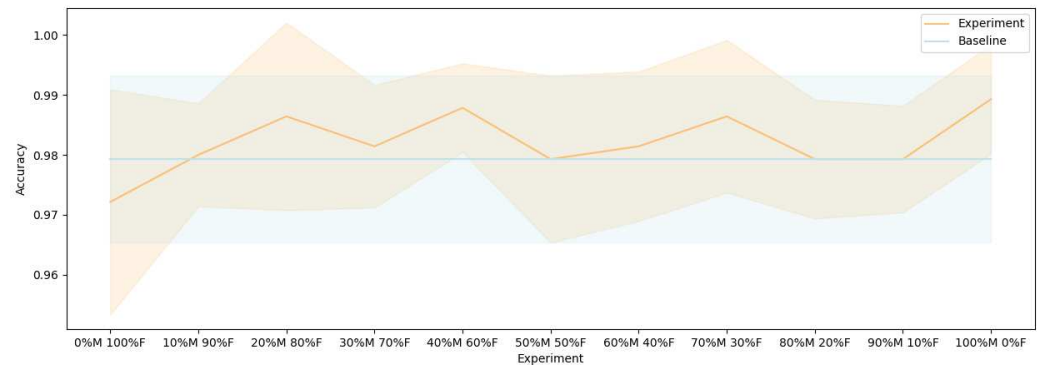

(b) Pneumonia VS COVID-19

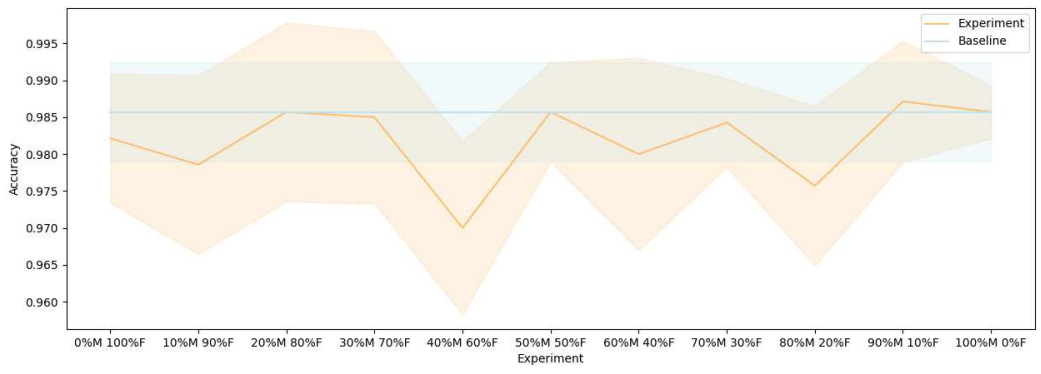

(c) Non-COVID-19 VS COVID-19

Figure 7: Mean \pm standard deviation test accuracy obtained for every studied scenario in every approach. 
good enough at every age range, and its standard deviation has a smaller peak at the older ages. Nevertheless, both the worsening in the obtained accuracy and the its instability are not of great magnitude in any approach. Thus, we can clearly observe in these graphs the clear tendency of the diagnosis offered to be influenced by age, regardless of the age group studied or the used computational approach. Moreover, it is noteworthy that this worsening is more or less present in all the cases studied, but is more pronounced in the older age groups, which is consistent given that the most critical cases of COVID-19 are more frequent in this group, resulting in a greater variability of pathological affectations in the lungs. For example, older patients are usually easily recognized by the wide range of different damaged ribcages they might present, being these caused by diseases or by the passing of time. In this situation, these patients are typically weaker in the face of such an aggressive disease as COVID-19, so different types of medical equipment, such as pathways or thoracostomy tubes, among other cardiac and pulmonary devices, are more present in these X-rays. All of these elements can appear on these images, obscuring lung densities typical of COVID-19 or leading our systems to recognise these patients more by the irregularity of their X-rays than by the signs of disease they may manifest, both affecting their COVID-19 diagnosis. However, these characteristics do not appear as frequently in the chest X-rays of younger patients, who typically have images where abnormalities are more easily observed and their association to COVID-19 is more straightforward, because they do not have other pathologies that may cause the presence of irregularities in their images. Hence, these reasons could justify the presence of this bias.

\section{Conclusions}

In this work, we have proposed the first study to analyze whether imbalance in chest $\mathrm{X}$-ray datasets produces biased deep learning approaches for COVID-19 screening with respect to the studied sex and age factors. For this purpose, 3 computational approaches using deep learning strategies that allowed us to carry out these studies of these factors in a detailed and comprehensive manner are presented and evaluated. To demonstrate the capabilities of our proposal, we perform several experiments on different public image datasets, including Normal, Pneumonia and COVID-19 cases. The presented results evidenced that the proposed methodology and tested approaches allow a robust and reliable analysis to support the clinical decisionmaking process in this pandemic scenario. Given the effort made to consider as many cases as possible and to make these studies as comprehensive as possible, we believe that the conclusions presented below are robust and reliable.

Regarding the sex-related imbalance analysis, we observed that this characteristic did not significantly affect the performance of our system. Whatever the sex ratio, the system performed well and provided satisfactory and stable results in all analyzed approaches. Since we performed a thorough study where we examined many different scenarios and explored different sex proportions, we can conclude that our system was not biased by this characteristic. Therefore, any difference observed between male and female patients from our dataset was not big enough to influence the system. On the other hand, regarding the age-related imbalance analysis, we observed that this characteristic did affect the performance of our system. It was clearly seen in every 


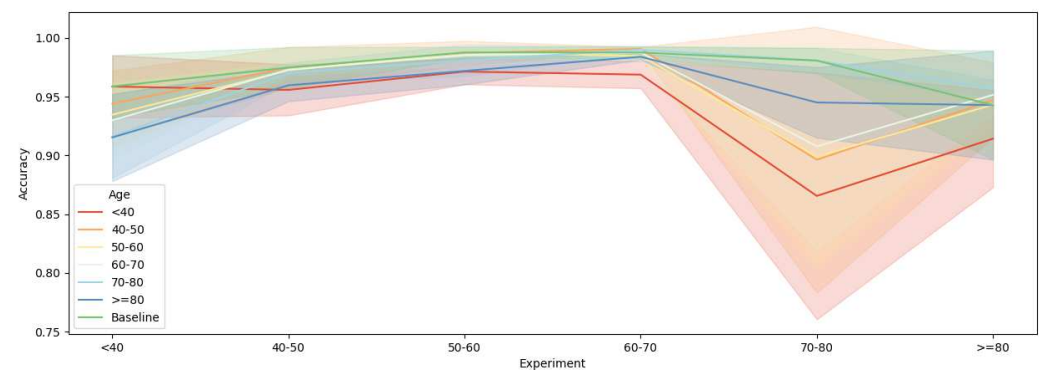

(a) Normal VS COVID-19

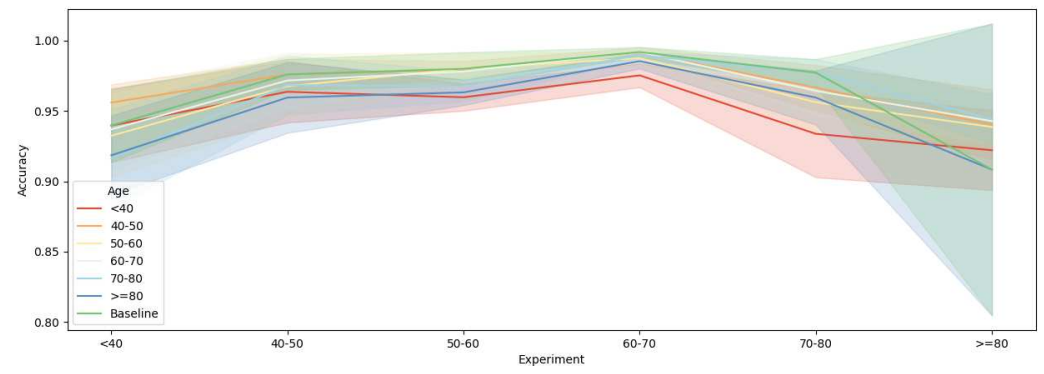

(b) Pneumonia VS COVID-19

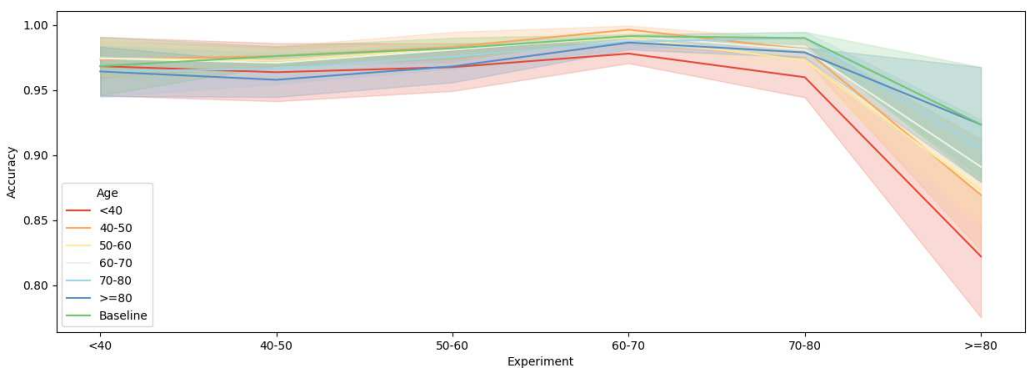

(c) Non-COVID-19 VS COVID-19

Figure 8: Mean \pm standard deviation test accuracy obtained for every studied age range in every approach. 
approach how the age used for training biased the system making it perform better for those with closer ages to the training phase one. Although obtained accuracy was good enough in every scenario as it was above $90 \%$ for most of the cases, age bias was consistent across all approaches. Again, since this analysis was conducted in a comprehensive manner, we can reliably conclude that the system was affected by the age of the patient. This could be caused by many reasons. For example, older patients have more irregular chest X-rays than younger people, since they can manifest different bone or cardiac pathologies. These differences might explain separability between the age ranges studied and their different results. Despite the fact a clear cause for this behaviour was not found, it is not necessary to emphasize how much it is needed to review the datasets being used for COVID-19 screening and identify possible bias related to the patient's age in them, since it was checked by our experiments that this factor's imbalance might affect the performance of the developed system.

As future work, it would be interesting to extend our study with patients diagnosed with other pulmonary disorders, such as emphysema, bronchitis and tuberculosis, among others. On the one hand, common pathologies affecting the lungs could represent a more challenging scenario of interest. On the other hand, expanding the dataset is of great interest to validate more completely the proposed methodologies. Other interesting future work would be to extend this analysis to other types of medical imaging modalities and correlate the results in a multimodal context to identify more precisely the influence of sex and age factors in COVID-19 screening systems.

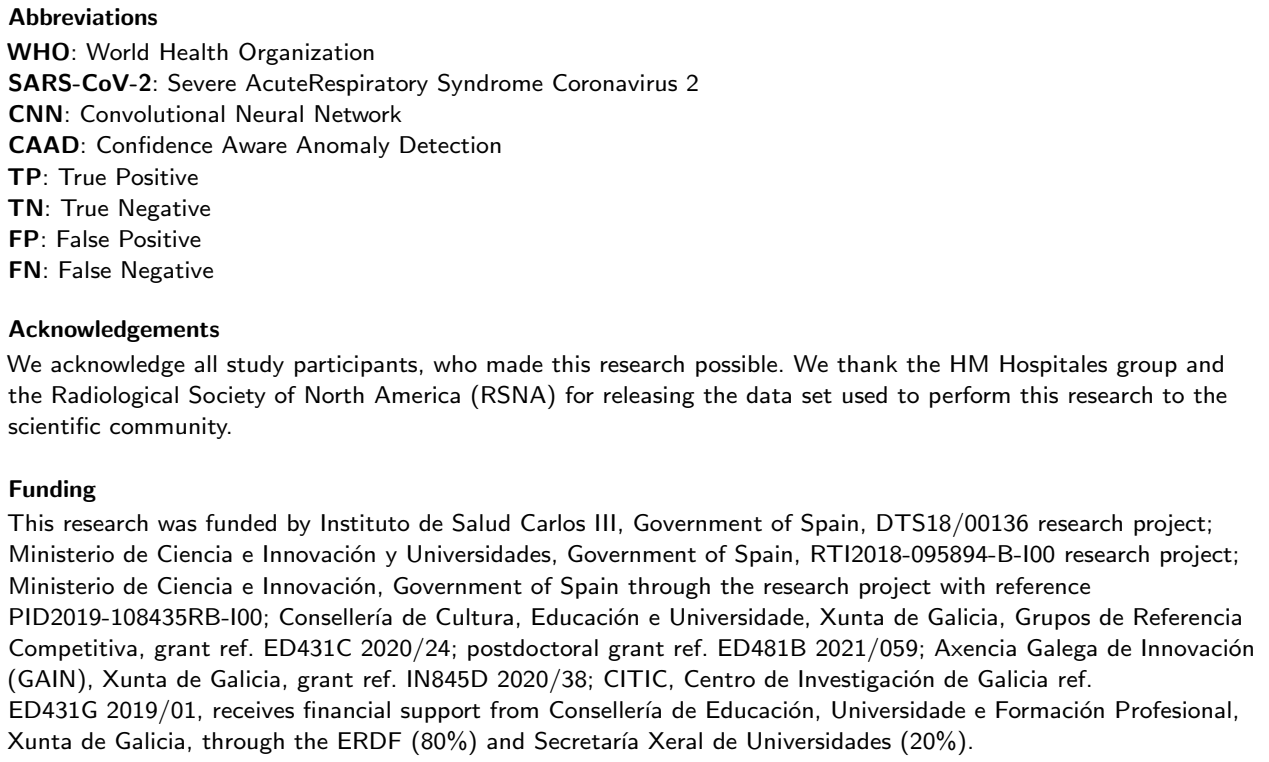

Ethics approval and consent to participate

The study was approved by the Ethics Review Board and Data Management Technical Commission of Galician Health Ministry for High Impact studies with protocol code 2020-007. In this study, all our data were obtained from publicly available datasets and we did not directly recruit or have direct contact with study participants. 
Availability of data and materials

The data that support the findings of this study are available from HM Hospitales group and the Radiological Society of North America (RSNA) but restrictions apply to the availability of these data, which were used under license for the current study, and so are not publicly available. Data are however available from the authors upon reasonable request and with permission of HM Hospitales group and the Radiological Society of North America (RSNA). The source code developed during this study is available from the corresponding author on reasonable request.

Competing interests

The authors declare that they have no competing interests.

Authors' contributions

Lorena Álvarez-Rodríguez: Conceptualization, Methodology, Software, Writing - Original Draft, Writing - Review \& Editing, Visualization. Joaquim de Moura: Conceptualization, Methodology, Software, Validation, Investigation, Writing - Review \& Editing, Supervision. Jorge Novo: Conceptualization, Methodology, Validation, Investigation, Writing - Review \& Editing, Supervision, Project administration. Marcos Ortega: Conceptualization, Methodology, Validation, Investigation, Writing - Review \& Editing, Supervision, Project administration, Funding Acquisition.

\section{Author details}

${ }^{1}$ Centro de Investigación CITIC, Universidade da Coruña, Campus de Elviña, 15071, A Coruña, Spain. ${ }^{2}$ Grupo VARPA, Instituto de Investigación Biomédica de A Coruña (INIBIC), Universidade da Coruña, 15006, A Coruña, Spain.

References

1. Serena Low, W.C., Chuah, J.H., Tee, C.A.T., Anis, S., Shoaib, M.A., Faisal, A., Khalil, A., Lai, K.W.: An overview of deep learning techniques on chest $\mathrm{x}$-ray and ct scan identification of covid-19. Computational and Mathematical Methods in Medicine 2021 (2021). doi:10.1155/2021/5528144

2. Mohammad-Rahimi, H., Nadimi, M., Ghalyanchi-Langeroudi, A., Taheri, M., Ghafouri-Fard, S.: Application of machine learning in diagnosis of covid-19 through $\mathrm{x}$-ray and ct images: a scoping review. Frontiers in cardiovascular medicine 8, 185 (2021). doi:10.3389/fcvm.2021.638011

3. Wang, L., Lin, Z.Q., Wong, A.: Covid-net: a tailored deep convolutional neural network design for detection of covid-19 cases from chest x-ray images. Scientific Reports 10(1), 19549 (2020). doi:10.1038/s41598-020-76550-z

4. Hammoudi, K., Benhabiles, H., Melkemi, M., Dornaika, F., Arganda-Carreras, I., Collard, D., Scherpereel, A.: Deep Learning on Chest X-ray Images to Detect and Evaluate Pneumonia Cases at the Era of COVID-19 (2020). 2004.03399

5. Hemdan, E.E.-D., Shouman, M.A., Karar, M.E.: COVIDX-Net: A Framework of Deep Learning Classifiers to Diagnose COVID-19 in X-Ray Images (2020). 2003.11055

6. Zhang, J., Xie, Y., Pang, G., Liao, Z., Verjans, J., Li, W., Sun, Z., He, J., Li, Y., Shen, C., et al.: Viral pneumonia screening on chest $\mathrm{x}$-rays using confidence-aware anomaly detection. IEEE transactions on medical imaging 40(3), 879-890 (2020)

7. Ozturk, T., Talo, M., Yildirim, E.A., Baloglu, U.B., Yildirim, O., Rajendra Acharya, U.: Automated detection of covid-19 cases using deep neural networks with x-ray images. Computers in Biology and Medicine 121, 103792 (2020). doi:10.1016/j.compbiomed.2020.103792

8. Shelke, A., Inamdar, M., Shah, V., Tiwari, A., Hussain, A., Chafekar, T., Mehendale, N.: Chest x-ray classification using deep learning for automated covid-19 screening. medRxiv (2020). doi:10.1101/2020.06.21.20136598.

https://www.medrxiv.org/content/early/2020/06/23/2020.06.21.20136598.full.pdf

9. Yoo, S.H., Geng, H., Chiu, T.L., Yu, S.K., Cho, D.C., Heo, J., Choi, M.S., Choi, I.H., Cung Van, C., Nhung, N.V., Min, B.J., Lee, H.: Deep learning-based decision-tree classifier for covid-19 diagnosis from chest x-ray imaging. Frontiers in Medicine 7, 427 (2020). doi:10.3389/fmed.2020.00427

10. Li, M.D., Arun, N.T., Gidwani, M., Chang, K., Deng, F., Little, B.P., Mendoza, D.P., Lang, M., Lee, S.I., O'Shea, A., et al.: Automated assessment of covid-19 pulmonary disease severity on chest radiographs using convolutional siamese neural networks (2020). doi:10.1101/2020.05.20.20108159

11. Chicco, D.: Siamese Neural Networks: An Overview, pp. 73-94. Springer, New York, NY" (2021)

12. de Moura, J., Novo, J., Ortega, M.: Fully automatic deep convolutional approaches for the analysis of covid-19 using chest x-ray images. medRxiv (2020). doi:10.1101/2020.05.01.20087254

13. Waheed, A., Goyal, M., Gupta, D., Khanna, A., Al-Turjman, F., Pinheiro, P.R.: Covidgan: Data augmentation using auxiliary classifier gan for improved covid-19 detection. IEEE Access 8, 91916-91923 (2020). doi:10.1109/ACCESS.2020.2994762

14. Morís, D.I., de Moura Ramos, J.J., Buján, J.N., Hortas, M.O.: Data augmentation approaches using cycle-consistent adversarial networks for improving covid-19 screening in portable chest $x$-ray images. Expert Systems with Applications 185, 115681 (2021). doi:10.1016/j.eswa.2021.115681

15. De Moura, J., García, L.R., Vidal, P.F.L., Cruz, M., López, L.A., Lopez, E.C., Novo, J., Ortega, M.: Deep convolutional approaches for the analysis of covid-19 using chest $x$-ray images from portable devices. IEEE Access 8, 195594-195607 (2020). doi:10.1109/ACCESS.2020.3033762

16. Mooney, P.: Chest x-ray images (Pneumonia). https://www .kaggle.com/paultimothymooney/chest-xray-pneumonia

17. Cirillo, D., Catuara-Solarz, S., Morey, C., Guney, E., Subirats, L., Mellino, S., Gigante, A., Valencia, A., Rementeria, M.J., Chadha, A.S., Mavridis, N.: Sex and gender differences and biases in artificial intelligence for biomedicine and healthcare. npj Digital Medicine 3(1) (2020). doi:10.1038/s41746-020-0288-5

18. Larrazabal, A.J., Nieto, N., Peterson, V., Milone, D.H., Ferrante, E.: Gender imbalance in medical imaging datasets produces biased classifiers for computer-aided diagnosis. Proceedings of the National Academy of Sciences 117(23), 12592-12594 (2020). doi:10.1073/pnas.1919012117 
19. Vidal, P.L., de Moura, J., Novo, J., Ortega, M.: Multi-stage transfer learning for lung segmentation using portable x-ray devices for patients with covid-19. Expert Systems with Applications 173, 114677 (2021). doi:10.1016/j.eswa.2021.114677

20. Covid Data Save Lives Dataset (2021). https://www.hmhospitales.com/coronavirus/covid-data-save-lives/english-version

21. of North America, R.S.: RSNA Pneumonia Detection Challenge (2018). https://www.rsna.org/education/a i-resources-and-training/ai-image-challenge/rsna-pneumonia-detection-challenge-2018

22. Wang, X., Peng, Y., Lu, L., Lu, Z., Bagheri, M., Summers, R.: Chestx-ray8: Hospital-scale chest X-ray database and benchmarks on weakly-supervised classification and localization of common thorax diseases. In: 2017 IEEE Conference on Computer Vision and Pattern Recognition(CVPR), pp. 3462-3471 (2017) doi:arxiv.org/abs/1705.02315

23. Huang, G., Liu, Z., van der Maaten, L., Weinberger, K.Q.: Densely connected convolutional networks. In: Proceedings of the IEEE Conference on Computer Vision and Pattern Recognition (2017). doi:arxiv.org/abs/1608.06993

24. Afifi, A., Hafsa, N.E., Ali, M.A.S., Alhumam, A., Alsalman, S.: An ensemble of global and local-attention based convolutional neural networks for covid-19 diagnosis on chest x-ray images. Symmetry 13(1) (2021). doi:10.3390/sym13010113

25. Wang, Z., Xiao, Y., Li, Y., Zhang, J., Lu, F., Hou, M., Liu, X.: Automatically discriminating and localizing covid-19 from community-acquired pneumonia on chest x-rays. Pattern Recognition 110, 107613 (2021). doi:10.1016/j.patcog.2020.107613

26. Minaee, S., Kafieh, R., Sonka, M., Yazdani, S., Jamalipour Soufi, G.: Deep-covid: Predicting covid-19 from chest $x$-ray images using deep transfer learning. Medical Image Analysis 65, 101794 (2020). doi:10.1016/j.media.2020.101794

27. Deng, J., Dong, W., Socher, R., Li, L.-J., Li, K., Fei-Fei, L.: Imagenet: A large-scale hierarchical image database. In: 2009 IEEE Conference on Computer Vision and Pattern Recognition, pp. 248-255 (2009). doi:10.1109/ACCESS.2021.3082638. IEEE

28. Ketkar, N.: Stochastic Gradient Descent, pp. 113-132. Springer, Berkeley, CA (2017)

29. Arias-Londoño, J.D., Gómez-García, J.A., Moro-Velázquez, L., Godino-Llorente, J.I.: Artificial intelligence applied to chest $\mathrm{x}$-ray images for the automatic detection of covid-19. a thoughtful evaluation approach. IEEE Access 8, 226811-226827 (2020). doi:10.1109/ACCESS.2020.3044858

30. Suri, J.S., Agarwal, S., Gupta, S.K., Puvvula, A., Biswas, M., Saba, L., Bit, A., Tandel, G.S., Agarwal, M. Patrick, A., Faa, G., Singh, I.M., Oberleitner, R., Turk, M., Chadha, P.S., Johri, A.M., Miguel Sanches, J., Khanna, N.N., Viskovic, K., Mavrogeni, S., Laird, J.R., Pareek, G., Miner, M., Sobel, D.W., Balestrieri, A., Sfikakis, P.P., Tsoulfas, G., Protogerou, A., Misra, D.P., Agarwal, V., Kitas, G.D., Ahluwalia, P., Teji, J., Al-Maini, M., Dhanjil, S.K., Sockalingam, M., Saxena, A., Nicolaides, A., Sharma, A., Rathore, V., Ajuluchukwu, J.N.A., Fatemi, M., Alizad, A., Viswanathan, V., Krishnan, P.K., Naidu, S.: A narrative review on characterization of acute respiratory distress syndrome in covid-19-infected lungs using artificial intelligence. Computers in Biology and Medicine 130, 104210 (2021). doi:10.1016/j.compbiomed.2021.104210 